EPJ Web of Conferences 13, 01001 (2011)

DOI: $10.1051 /$ epjconf/20111301001

(c) Owned by the authors, published by EDP Sciences, 2011

\title{
Jet Tomography versus Holography at RHIC and LHC
}

\author{
M. Gyulassy,a , A. Buzzatti, A. Ficnar, J. Noronha, and G. Torrieri \\ Department for Physics, Columbia University, Pupin Lab, New York NY 10027
}

\begin{abstract}
We compare pQCD based jet tomography to AdS/CFT based jet holography approach to address the heavy quark jet puzzle and discuss future tests at RHIC and LHC that could help decide which paradigm can provide the most consistent quantitative theory to explain modification of jet observabkles in high energy nuclear collisions.
\end{abstract}

\section{The Heavy Quark Puzzle}

The experimental discovery [1] of a strongly coupled and approximately conformal invariant phase of Quark Gluon Plasmas (sQGP) [2] at the Relativistic Nuclear Collider (RHIC) using $\mathrm{Au}+\mathrm{Au}$ reactions at $\mathrm{cm}$ energies $200 \mathrm{AGeV}$ (100 times the sum of the nuclear rest mass) has generated wide interest. The new phase exhibits several key dynamical properties (especially, minimally dissipative "perfect" fluidity, and high opacity and quenching of jet probes) that have defied a satisfactory explanation in terms perturbative Quantum Chromodynamics (pQCD) based dynamical models - so far. This has led many theorists to abandon QCD and to jump into a fifth dimensional gedanken black hole located in the depths of an Anti de Sitter $\left(\mathrm{AdS}_{5}\right)$ gravity world conjectured by Maldacena and others[8] to provide a dual "holographic" geometric description of very strongly coupled supersymmetric gauge theories in a special t'Hooft $\left(N_{c} \rightarrow \infty \lambda_{t H}=g^{2} N_{c} \rightarrow \infty\right)$ limit. See Fig. 1 from [9].

One of the most surprising and unexpected properties of the sQGP phase that has driven some to this radical departure from the conventional standard model paradigm is the very high opacity of the sQGP to even highest energy and heaviest bottom quark jets. Recently, however, a more refined pQCD based theory of radiative energy loss has been developed by Djordjevic et al[3] that question the need to jump into $\mathrm{AdS}_{5}$ black holes just yet. Previously neglected chromo magnetic interactions and finite size nonstatic coherence effects in perturbative QGPs were shown to increase the radiative energy loss of heavy quarks by up to a factor of two, possibly solving at least part of the current heavy quark jet puzzle.

The sQGP produced in $\mathrm{Au}+\mathrm{Au}$ reactions at $\mathrm{RHIC} / \mathrm{BNL}$ (0.2 ATeV) and since November 2010 in $\mathrm{Pb}+\mathrm{Pb}$ collisions at LHC/CERN (5.5 ATeV) energies exits for only fleeting $\Delta t \sim 10 \mathrm{Fermi} / \mathrm{c}$ ( few $10^{-23} \mathrm{sec}$ ) and produced in a cylindrical volume of transverse radius only $L \sim 5$ Fermis $\left(<10^{-12} \mathrm{~cm}\right)$. Yet it appears to reach near perfect local equilibrium at temperatures $T \sim 300-500 \mathrm{MeV}$

\footnotetext{
a e-mail: gyulassy@phys.columbia.edu
}

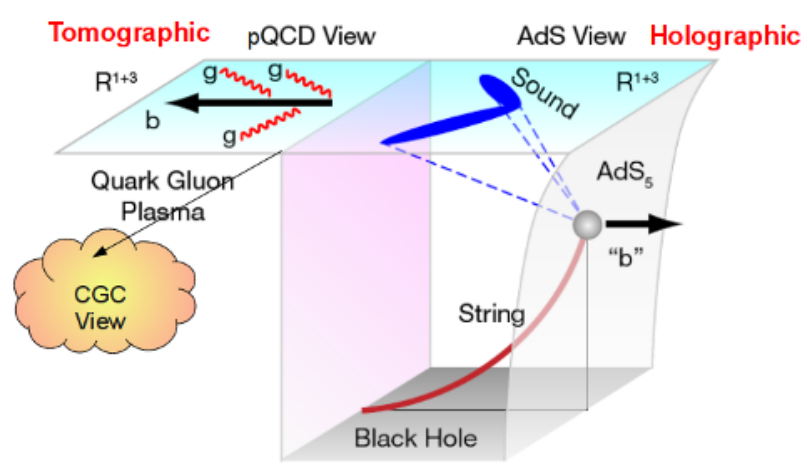

Fig. 1. Schematic comparison of jet tomography versus tomography (modified figure from [9]). The first (on left side) is based on standard quantum multiple collision radiative and elastic pQCD Feynman amplitudes. The QGP plasma is modeled as a thermal Hard Thermal Loop gas of dynamically screened quarks and gluon quasipartons. A jet is the standard model off-shell high $p_{T}$ parton. The holography view (right side) is based on a conjectured classical gravity/string dual description in terms of dynamics of strings in an $\mathrm{AdS}_{5}$ background. The QGP plasma is modeled as the 4D hologram of a Black Hole (BH) at a fixed AdS depth proportional to the Hawking temperature. A "jet" is modeled $\mathrm{d}$ as a string dragging over the $\mathrm{BH}$ at a fixed height proportional to it rest Mass. Energy by radiating gravitons cause Mach like stress ripples on the 4D boundary. At LHC initial state suppression of hard probes by the Color Glass Condensate gluon saturation phenomena complicate (cloud in foreground) the final state tomographic analysis of $\mathrm{PbPb}$ reactions. Furure $\mathrm{p}+\mathrm{Pb}$ control experiments will be required to determine experimentally the magnitude of initial state (CGC) effects.

(few $10^{12} \mathrm{~K}$ ). From the asymptotically free QCD point of view, the QGP phase was predicted over thirty years ago to exist as a novel deconfined, chiral symmetric, and approximately conformal invariant phase of QCD matter when temperatures exceed a critical temperature $T_{c} \approx 200$ $\mathrm{MeV}$. This phase differs drastically from the well studied low temperature $T<T_{c}$ hot nuclear/hadron resonance gas phase where all the quarks and gluons are confined into 
color neutral baryons and mesons and both the chiral and conformal symmetry of the QCD action is manifestly broken. Numerical nonperturbative lattice QCD calculations confirm that for $T$ above a few times $T_{c}$, the pressure $P(T)$ (equation state) of a sQGP differs by only $\sim 20 \%$ from an ideal Stefan Boltzmann (SB) gas of massless, deconfined quarks and gluons.

The idea of jet tomography[4] is to use the observed attenuation pattern (the nuclear modification of the $\mathrm{A}+\mathrm{A}$ jet yield relative to binary collision scaled $p+p$ jet yields) as a way to measure the energy loss per unit length, $d E / d x$, as a function of jet energy, mass and path length. Given a theory of quark energy loss, tomographic properties of the medium can be inferred similar to how computer aided tomography with X-rays or positrons are used routinely in medical applications. An important feature of bottom jets is that their initial flux distribution can be experimentally calibrated $p+p$ and checked against NLO pQCD theory. In addition, deuterium beam control experiments $D+A \rightarrow b \bar{b}+X$ can be used to deconvolute initial state nuclear effects.

In Ref. [6] DGLV generalized the GLV theory [5] to all orders in opacity theory for heavy quarks. The DGLV theory predicted that bottom quark jets should be much less quenched than light mass jets. However, non-photonic single electron data from RHIC revealed [7] that bottom quarks must be attenuated as much as light quark, gluon, or charm jets. The data indicated that only $30 \%$ of the produced bottom jets at $p_{T} \sim 10-20 \mathrm{GeV}$ survived passing through the highly opaque sQGP medium. Subsequent attempts to improve the theory [11] by including finite opacity fluctuations elastic as well as inelastic energy loss and fluctuations of path lengths could reduce but not eliminate the $\mathrm{pQCD}$ predicted weaker attenuation discrepancy with single electron data.

In 2006 a radically new idea was proposed by Gubser and Herzog et al[12] based on the conjectured AdS/CFT holography paradigm. In that picture heavy quark jet propagation in a SQGP is assumed to be dual to the problem of string drag between a D7 "support" probe brane and a D3 black brane that serves as a geometric model of the sQGP phase of an analogous $\mathcal{N}=4$ super-symmetric Yang Mills cousin of QCD. This geometric string drag setup in in a background $A d S_{5} \times S_{5}$ geometry is pictured very schematically in Fig. 1 . At infinite 'Hooft coupling of course even a stampeding elephant would be stopped. However, we recent found [13] that this holographic model can simultaneously explain of both hard and soft sQGP dynamical observables when extrapolated down to more realistic $N_{c}=3$ and $\lambda_{t H} \sim 20$. See later section.

The above successes of AdS holography of course does not exclude that a sufficiently refined $\mathrm{pQCD}$ based approach could be developed to explain the data. This is what we show in the next section. Duality implies an equivalence of descriptions in different terms and computational algorithms. It is generally more difficult to compute in one description than in the other in any particular situation, but both address are assumed to be able in principle to describe the same physics. The extended kinematic range and powerful detector capabilities at the LHC will soon allow new robust jet observables $[14,15]$ to help further discriminate between competing/complementary QCD and AdS holographic paradigms.

\section{2 pQCD Jet Tomography}

Fig. 2 summarizes the master formula from DGLV[6] for the medium induced gluon invariant number distribution at arbitrary order in opacity $\left(L / \lambda_{q}\right)^{n}$.

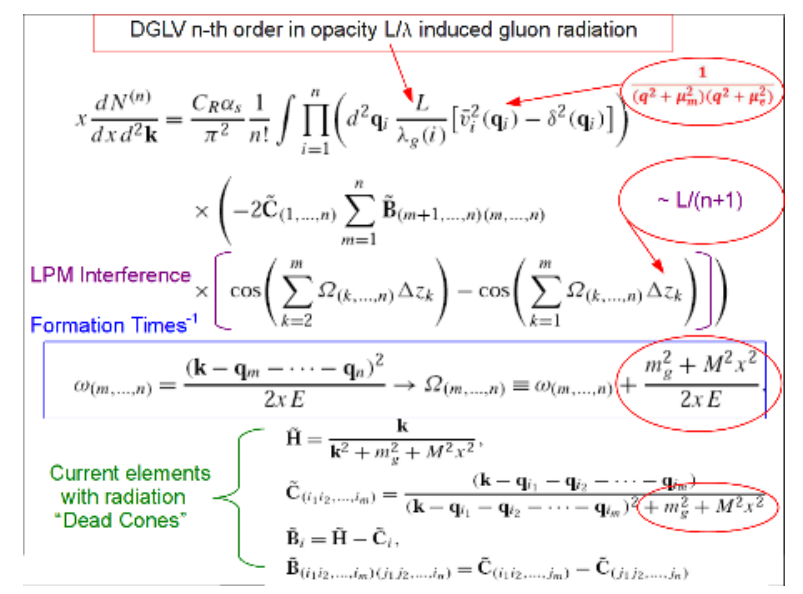

Fig. 2. Radiative energy loss double differential distribution within the DGLV [6] formalism is illustrated at arbitrary order on opacity. Key input elements are the transverse momentum, $\mathbf{q}_{\mathbf{i}}$, kick distributions $\bar{v}_{i}\left(\mathbf{q}_{\mathbf{i}}\right.$ along the jet path the path. The scattering center longitudinal distance separations, $\Delta z_{i}$ together local formations times $\left.1 / \Omega_{(} m \cdots n\right)$ control the destructive LPM interference in finite size systems limited to $L<10 \mathrm{fm}$. The quark mass $M$ leads to a dead cone effect that reduces bottom quark energy loss to light quark jet loss. Recent progress is due to the development of new Monte Carlo numerical codes to compute high orders in the opacity expansion. Please refer to [6] for details.

In the dynamic modified MDGLV [16] generalization of GDLV the (static Debye screened) momentum distributions in the GLV(eq.113)/DGLV(eq.17) multiple collision kernel is replaced with a path dependent effective magnetic enhanced transverse distributions

$$
\bar{v}^{2}\left(z, \mathbf{q} ; r_{m}\right)=\frac{\mu_{e}(z)^{2} \mathcal{N}\left(r_{m}\right)}{\pi} \frac{1}{\left(\left(\mathbf{q}^{2}+\mu_{e}^{2}(z)\right)\right)\left(\left(\mathbf{q}^{2}+r_{m}^{2} \mu_{e}^{2}(z)\right)\right)},
$$

here $0 \leq r_{m} \equiv \mu_{m} / \mu_{e} \leq 1$ is the ratio of the color electric Debye and the assumed longer color magnetic screening lengths. At higher than first order in opacity orders the unitarity corrections diverge in the (unphysical) $r_{m}=0$ limit and require special care. Fortunately, in the finite size quark gluon plasma produced in $\mathrm{A}+\mathrm{A}, r_{m}$ is bounded from below by $1 /\left(\mu_{e} R_{A}\right)$ due to confinement of color outside the finite size plasma. We consider here finite $r_{m} \geq 1 / 3$ to explore the sensitivity of the bottom to charm jet ratio to enhanced soft momentum transfer due to perturbative magnetic field fluctuations of range up to $3 / g T$. See Fig 3 . 
The left panel of Fig. 3 shows the ratio of light (up) quark energy loss to bottom quark energy vs path length $2 \leq L \leq 4 \mathrm{fm}$ for different $r_{m}$ and different orders in opacity up to third order. It is found that charm and up quarks have essentially identical energy loss. What is clear is that neither long range magnetic scattering nor higher orders are sufficient to bring the ratio of light to heavy quark jet energy loss near unity for $L>2 \mathrm{fm}$. Despite the fact that the absolute value of $\triangle E(Q)$ is almost doubled by switching from the pure static $r_{m}=1$ to the dynamical $r_{m}=0$ model in the uniform brick geometry the $\mathrm{c} / \mathrm{b}$ and $\mathrm{u} / \mathrm{b}$ energy loss ratios are surprising insensitive to the magnetic screening length.
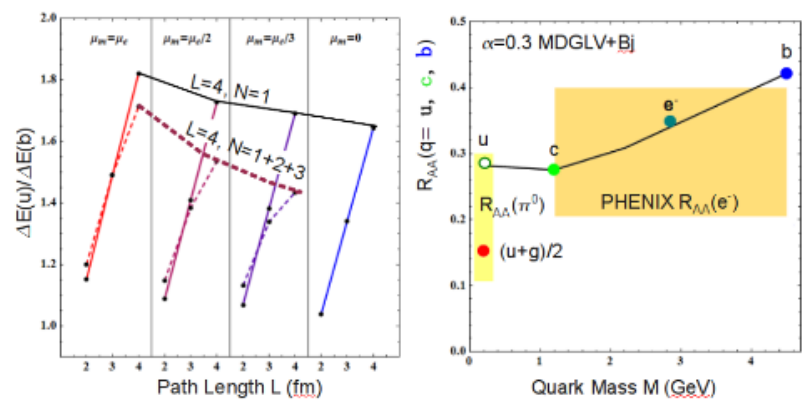

Fig. 3. The sensitivity of the radiated energy loss for light (u,d,s,c) quark jets compared to heavy bottom quark jets as a function of the path length $L=2-4 \mathrm{fm}$ in a uniform pQCD plasma with static Debye screening mass $\mu_{e}=0.5 \mathrm{fm}$ and variable magnetic mass $\mu_{m} / \mu_{e}=0,1.3,1.2,1$ from [16]. Lowest order $\mathrm{N}=1$ compared to up to third oder in opacity is also compared. Dynamic magnetic scattering does not significnatly reduce the ratio of up/bottom energy loss. Hence as shown on the right panel the nuclear modification factor ration between bottom and charm quarks does not come close to unity even in the ideal HTL limit $\mu_{m}=0$ denoted by MDGLV. The very large uncertainty in present PHENIX data for the electron energy range $p_{T}=5.5-8.5 \mathrm{GeV}$ focused on here precludes a more definitive experimental test of the pQCD tomographic prediction. Future flavor tagged jet studies resolving charm and bottom jets are needed to establish if MDGLV+Bj can quantitatively account light pion $R_{A A}(p i)$ with future heavy quark $R_{A A}(c)$ and $R_{A A}(b)$ data with common pQCD plasma parameters.

On the right panel of Fig. $3, R_{A A}(Q)$ is shown after adding the average WHDG elastic energy loss contribution. The shadowed regions represent the current rather large error bars in the pion and nonphotonic electron data. If we take into account the large error bands in both the pion and the nonphotonic electron data then including color magnetic effects in diffuse expanding geometries do help to reduce the significance of the heavy quark puzzle as posed in WHDG. In this representation the "Jet Flavor Spectroscopy" hierarchy predicted in the pQCD framework including dynamical scattering effects as well as dynamical expanding geometry:

$$
R_{A A}(g)<R_{A A}(\pi)<R_{A A}(c)<R_{A A}\left(e^{-}\right)<R_{A A}(b)<R_{A A}(\gamma)
$$

is actually not be incompatible with present large error bands for both light and heavy jet tomography. Clearly, future reduction of the error bands can test the remaining $50 \%$ enhancement bottom to charm nuclear modification.

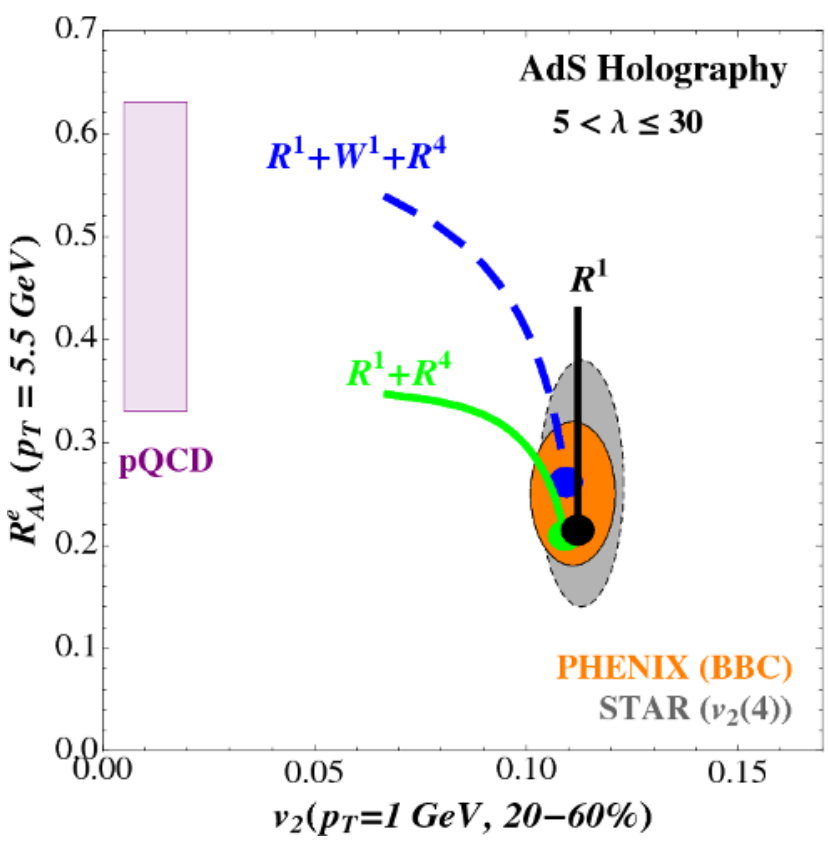

Fig. 4. Conformal holography (from [13]) showing the correlation between the high $p_{T} \sim 10 \mathrm{GeV}$ heavy quark jet nuclear modification in central $\mathrm{Au}+\mathrm{Au}$ (as measured via nonphotonic electrons) and bulk plasma elliptic flow in noncentral reactions (as measured by the second azimuthal harmonic of low $p_{T}$ hadrons. WHDG and especially dynamically improved MDGLV tomography shown in Fig. 3 can come within error band of $R_{A A}^{e}$ data, but pQCD fails to account for the low viscosity of the sQGP required to explain the observed elliptic harmonic. The analytic predictions of conformal holography for both the heavy quark drag and the viscosity to entropy ratio were used to generate the black, green and dashed blue curves. The black curve is for the idealized Hilbert action that fits the hard/soft correlation datum point when $\lambda_{\text {tHooft }} \sim 20$. The green curve shows the effect of 4 th order in curvature corrections that are found negligible for the strong coupling $\lambda_{\text {tHooft }} \sim 20$ range prefered by the datum. The blue dashed include also estimated world sheet fluctuation corrections that tend to reduce heavy quark energy loss, denoted by $W^{1} \sim O\left(1 / \sqrt{\lambda_{t H o o f t}}\right.$. The robustness of the conformal holographic prediction with one parameter $\lambda_{t \text { Hooft }} \sim 20$ explaining both bulk elliptic flow and heavy quark jet quenching is truly remarkable.

\section{Conformal Holography}

Conformal holography refers to the predicted temperature independence of $s / s_{S B}$, the entropy density of a very strongly coupled SYM compared to its ideal Stefan Boltzmann limit, and also of the viscosity to entropy density ratio. The heavy quark jet relaxation rate, $1 / \tau_{Q}$, is controlled by the 
$\mu_{Q}=\sqrt{\lambda} \pi T^{2} / 2 M_{Q}$ for a heavy quark with mass $M_{Q}$ in a plasma of temperature $T$ [12]. The relaxation time is related to the heavy quark energy loss per unit length through $\tau_{Q}(\lambda)=-1 /(d \log p / d t)=-1 /(d \log E / d x)$, where $p=$ $M_{Q} \gamma v$ and $v=p / E$.

The analysis in [13] is based on the following remarkably simple algebraic expressions relating three fundamental nonperturbative properties of large $N_{c}, \mathcal{N}=4$ conformal SYM plasmas at large t'Hooft coupling $\lambda$ :

$$
\begin{aligned}
\frac{s}{s_{S B}} & =\frac{3}{4}\left(1+\frac{c_{3}}{8 \lambda^{3 / 2}}\right), \\
\frac{\eta}{s} & =\frac{1}{4 \pi}\left(1+\frac{c_{3}}{\lambda^{3 / 2}}\right), \\
\tau_{Q}^{-1} & =\mu_{Q}\left(1+\frac{\kappa_{1}}{\lambda^{1 / 2}}+\frac{c_{3}}{16 \lambda^{3 / 2}}\right)
\end{aligned}
$$

where $c_{3}=15 \zeta(3) \approx 18, \kappa_{1} \sim-1$ is a new worldsheet fluctuation correction amplitude discussed below. The $\lambda^{-3 / 2}$ correction to the entropy density ratio in Eq. (2) was found in [17] while the analogous correction to $\eta / s$ was obtained from [18]. The finite t'Hooft coupling correction to the heavy quark energy loss, $c_{3} \lambda^{3 / 2} / 16$, is a new result reported in [13] and is needed for a consistent application of the strongly-coupled $\mathcal{N}=4 \mathrm{SYM}$ model to heavy ion reactions. As shown in Fig. 4, conformal holography provides a remarkable robust solution not only for the heavy quark puzzle but also the near perfect fluidity of the sQGP (as determined by the second elliptic Fourier harmonic, $v_{2}\left(p_{T} \sim 1 \mathrm{GeV}\right)$, of the global azimuthal distribution in noncentral collisions.

However, as shown in Ref. [13] conformal holography makes a very strong and hence easily falsifiable prediction. It predicts that $R_{A A}(b) / R_{A A}(c) \sim 4$. This confirms earlier predictions from [14] that this double ratio will be one of the most decisive observables between competing paradigms once identified heavy flavor tagged jet quenching is measured.

\section{Nonconformal Holography of heavy quark energy loss}

One of the key defects of conformal holography is its failure to account to the QCD confinement transition below $T_{c} \sim 160 \mathrm{MeV}$ into a viscous hadronic resonance gas. It applies at best to observables dominated by strongly coupled dynamics above the deconfinement temperature.

In order to improve this defect, recent phenomenological additions to the Hilbert action involing dilaton fields and potentials was proposed in [19]. In [20] we used a sixed order dilaton potential to fit the lattice QCD data on the conformal anomaly as well as the Polyakov loop and speed of sound in the interesting QCD transition temperature region. The dilaton potential breaks conformal invariance in a way consistent with known QCD thermodynamics. With the nonconformal metric so constrained, we then computed in the generalized string drag energy loss derived in [21]. Fig. 5. shows our first estimate of the nontrivial temperature dependence of the ratio of the charm and bottom energy loss. At high temperatures, where approximate conformal invariance is recovered, this ratio approaches the large ratio $\sim 3.5$ in the conformal holographic limit. However, near $T_{c}$ the ratio reduces toward $\sim 1.5$. Whether this QCD constrained nonconformal holographic extension solves the heavy quark puzzle without introducing new puzzles remains to be seen.

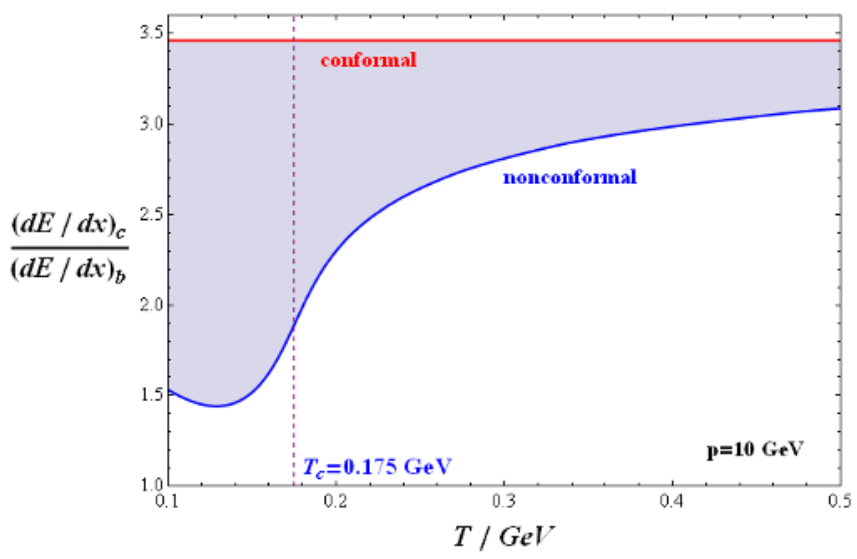

Fig. 5. Preliminary results from a phenomenological noconformal extendion of AdS holography via a dilaton potential adjusted to fit the lattice QCD data on the temperature dependence of the entropy density, the conformal anomaly, and Polyakov loop through the cross over transition near $T_{c}=175 \mathrm{MeV}$. The ratio of $d E / d x$ of $\mathrm{c}$ to $\mathrm{b}$ quarks is found to decrease drastically from 3 in the approximately conformal high temperature range $T \sim 300$ $\mathrm{MeV}$ to about 1.5 just below $T_{c}$. This nonconformal holographic model reduces significantly difference between $\mathrm{pQCD}$ tomography and conformal holography.

\section{References}

1. BNL -73847-2005 Formal Report; Nucl.Phys.A757,pp1, 28, 102,184 (2005).

2. M. Gyulassy and L. McLerran, Nucl. Phys. A 750, 30 (2005).

3. M. Djordjevic, Phys. Rev. C80, 064909 (2009); Nucl. Phys. A 830, 163C (2009).

4. M. Gyulassy and M. Plumer, Phys. Lett. B 243, 432 (1990). X. N. Wang and M. Gyulassy, Phys. Rev. Lett. 68, 1480 (1992);

5. M. Gyulassy, P. Levai and I. Vitev, Nucl. Phys. B 594, 371 (2001); I. Vitev and M. Gyulassy, Phys. Rev. Lett. 89, 252301 (2002);

6. M. Djordjevic et al Nucl. Phys. A 733, 265 (2004); Phys. Rev. Lett. 94, 112301 (2005)

7. S. S. Adler et al. [PHENIX Collaboration], Phys. Rev. Lett. 96, 032301 (2006), ibid 98, 172301 (2007); B. I. Abelev et al. [STAR Collaboration], Phys. Rev. Lett. 98, 192301 (2007); 
8. J. M. Maldacena, Adv. Theor. Math. Phys. 2, 231 (1998) [Int. J. Theor. Phys. 38, 1113 (1999)]; E. Witten, Adv. Theor. Math. Phys. 2, 253 (1998).

9. M. Gyulassy, "Getting to the Bottom of the Heavy Quark Jet Puzzle”, APS Physics 2, 107 (2009).

10. M. Djordjevic et al, Phys. Lett. B 632, 81 (2006)

11. S. Wicks et alNucl. Phys. A 784, 426 (2007).

12. C. P. Herzog et al,JHEP 0607, 013 (2006); S. S. Gubser, Phys. Rev. D 74, 126005 (2006).

13. J.Noronha, M. Gyulassy,G. Torrieri, Phys. Rev. C82, 054903 (2010).

14. W. A. Horowitz and M. Gyulassy, arXiv:0706.2336 [nucl-th].

15. B. Betz et al Phys. Lett. B 675, 340 (2009)

16. A. Buzzatti, M. Gyulassy, [arXiv:1012.0614 [hep-ph]].

17. S. S. Gubser, I. R. Klebanov and A. A. Tseytlin, Nucl. Phys. B 534, 202 (1998).

18. R. C. Myers, et al Phys. Rev. D 79, 041901 (2009); A. Buchel, et al Phys. Lett. B 669, 364 (2008).

19. S. S. Gubser and A. Nellore, Phys. Rev. D 78, 086007 (2008), arXiv:0804.0434v1 [hep-th]

20. A. Ficnar, J. Noronha, M. Gyulassy, "Nonconformal Holography of Heavy Quark Quenching," [arXiv:1012.0116 [hep-ph]].

21. C. P. Herzog, JHEP 0609, 032 (2006), arXiv:hepth/0605191v2

22. A.Buzzatti, A. Ficnar, S. Wicks, M. Gyulassy, DGLVBFW-MC1.0 opacity series code, in preparation. S. Wicks, arXiv:0804.4704 [nucl-th]. 